PROCEEDINGS OF THE

AMERICAN MATHEMATICAL SOCIETY

Volume 128, Number 12, Pages 3523-3528

S 0002-9939(00)05439-3

Article electronically published on June 7, 2000

\title{
PROJECTIVE BOUNDEDNESS AND CONVOLUTION OF FRÉCHET MEASURES
}

\author{
R. BLEI AND J. CAGGIANO
}

(Communicated by Christopher D. Sogge)

\begin{abstract}
Fréchet measures of order $n$ ( $\mathcal{F}_{n}$-measures $)$ are the measuretheoretic analogues of bounded $n$-linear forms on products of $C_{0}(K)$ spaces. In an LCA setting, convolution of $\mathcal{F}_{2}$-measures is always defined, while there exist $\mathcal{F}_{3}$-measures whose convolution cannot be defined. In a three-dimensional setting, we demonstrate the existence of an $\mathcal{F}_{2}$-measure which cannot be convolved with arbitrary $\mathcal{F}_{3}$-measures.
\end{abstract}

Let $\left(\mathcal{X}_{1}, \mathcal{A}_{1}\right), \ldots,\left(\mathcal{X}_{n}, \mathcal{A}_{n}\right)$ be measurable spaces. A scalar-valued function $\mu$ on $\mathcal{A}_{1} \times \cdots \times \mathcal{A}_{n}$ is a Fréchet measure (an $\mathcal{F}_{n}$-measure) if, when any $n-1$ coordinates are fixed, $\mu$ is a measure in the remaining coordinate. When the measure spaces are arbitrary or understood, we write $\mathcal{F}_{n}$ for $\mathcal{F}_{n}\left(\mathcal{A}_{1}, \ldots, \mathcal{A}_{n}\right)$. Let $\mathcal{X}_{1}, \ldots, \mathcal{X}_{n}$ be locally compact abelian groups with corresponding dual groups $\hat{\mathcal{X}}_{1}, \ldots, \hat{\mathcal{X}}_{n}$. If $\mathcal{A}_{1}, \ldots, \mathcal{A}_{n}$ are the respective Borel fields of $\mathcal{X}_{1}, \ldots, \mathcal{X}_{n}$ and $\mu \in \mathcal{F}_{n}$, then the Fourier-Stieltjes transform of $\mu$ is given by

$$
\hat{\mu}\left(\gamma_{1}, \ldots, \gamma_{n}\right)=\int \bar{\gamma}_{1} \otimes \cdots \otimes \bar{\gamma}_{n} d \mu, \quad\left(\gamma_{1}, \ldots, \gamma_{n}\right) \in \hat{\mathcal{X}}_{1} \times \cdots \times \hat{\mathcal{X}}_{n} .
$$

(The integral above is defined iteratively.) We use the multi-linear Riesz Representation Theorem [B1] to identify $\mathcal{F}_{n}\left(\mathcal{A}_{1}, \ldots, \mathcal{A}_{n}\right)$ with the dual space of

$$
C_{0}\left(\mathcal{X}_{1}\right) \hat{\otimes} \cdots \hat{\otimes} C_{0}\left(\mathcal{X}_{n}\right) \equiv \mathcal{V}_{n}\left(\mathcal{X}_{1}, \ldots, \mathcal{X}_{n}\right),
$$

and thus extend the action to arbitrary bounded functions by integration. See [B3] for details. It is natural to consider the feasibility of convolution of $\mathcal{F}_{n}$-measures.

Definition 1. Let $\mathcal{X}_{1}, \ldots, \mathcal{X}_{n}$ be LCA groups with Borel fields $\mathcal{A}_{1}, \ldots, \mathcal{A}_{n} . \mathcal{F}_{n^{-}}$ measures $\mu$ and $\nu$ are convolvable if $\hat{\mu} \hat{\nu}=\hat{\lambda}$ for some $\lambda \in \mathcal{F}_{n} ; \lambda$ is then denoted by $\mu * \nu$ or $\nu * \mu$. We say $\mu \in \mathcal{F}_{n}$ is a convolver if $\mu * \nu$ exists for all $\nu \in \mathcal{F}_{n}$.

The case $n=1$ in Definition 1 is classical; every $\mathcal{F}_{1}$-measure is a convolver. It is shown in GS1 that in a two-dimensional setting every $\mathcal{F}_{2}$-measure is a convolver, while in GS2 it is shown that there exist non-convolvers in $\mathcal{F}_{3}$. In general, convolvability is related to projective boundedness, a property conveying a Grothendieck-type inequality. In the definition which follows, $\mathcal{L}^{\infty}\left(\mathcal{X}_{j}\right)$ denotes the space of bounded functions on $\mathcal{X}_{j}$.

Received by the editors September 1, 1998 and, in revised form, January 28, 1999.

1991 Mathematics Subject Classification. Primary 43A05, 46A32.

The first author was supported by an NSA grant. 
Definition 2 (B3] $)$. Let $\left(\mathcal{X}_{1}, \mathcal{A}_{1}\right), \ldots,\left(\mathcal{X}_{n}, \mathcal{A}_{n}\right)$ be measurable spaces, and let $\mu \in$ $\mathcal{F}_{n}\left(\mathcal{X}_{1}, \ldots, \mathcal{X}_{n}\right)$. For $f_{j} \in \mathcal{L}^{\infty}\left(\mathcal{X}_{j}\right), j=1, \ldots, n$, define

$$
\phi_{\mu}\left(f_{1}, \ldots, f_{n}\right)=\int f_{1} \otimes \cdots \otimes f_{n} d \mu .
$$

We say that $\mu$ is projectively bounded if

$$
\begin{aligned}
\|\mu\|_{p b_{n}}:=\sup \left\{\left\|\left.\phi_{\mu}\right|_{E_{1} \times \cdots \times E_{n}}\right\| \|_{\mathcal{V}_{n}\left(E_{1}, \ldots, E_{n}\right)}:\right. & \\
& \left.E_{j} \subset \operatorname{Ball}\left(\mathcal{L}^{\infty}\left(X_{j}\right)\right),\left|E_{j}\right|<\infty, j=1, \ldots, n\right\}<\infty ;
\end{aligned}
$$

the class of projectively bounded $\mathcal{F}_{n}$-measures on $\mathcal{X}_{1} \times \cdots \times \mathcal{X}_{n}$ is denoted by $\mathcal{P B F}_{n}=\mathcal{P B F}_{n}\left(\mathcal{X}_{1}, \ldots, \mathcal{X}_{n}\right)$.

Let $\mathcal{X}_{1}, \ldots, \mathcal{X}_{n}, \mathcal{Y}_{1}, \ldots, \mathcal{Y}_{n}$ be locally compact Hausdorff spaces with respective Borel fields $\mathcal{A}_{1}, \ldots, \mathcal{A}_{n}, \mathcal{B}_{1}, \ldots, \mathcal{B}_{n}$. For

$$
f \in \mathcal{V}_{n}\left(\mathcal{X}_{1} \times \mathcal{Y}_{1}, \ldots, \mathcal{X}_{n} \times \mathcal{Y}_{n}\right) \quad \text { and } \quad \mu \in \mathcal{F}_{n}\left(\mathcal{A}_{1}, \ldots, \mathcal{A}_{n}\right)
$$

define a function $\eta_{f ; \mu}$ on $\mathcal{Y}_{1} \times \cdots \times \mathcal{Y}_{n}$ by

$$
\eta_{f ; \mu}\left(y_{1}, \ldots, y_{n}\right)=\int f\left(x_{1}, y_{1}, \ldots, x_{n}, y_{n}\right) \mu\left(d x_{1}, \ldots, d x_{n}\right) \quad\left(y_{j} \in \mathcal{Y}_{j}\right) .
$$

Definition 3. Let $\mu \in \mathcal{F}_{n}\left(\mathcal{A}_{1}, \ldots, \mathcal{A}_{n}\right)$.

1. ( $\tau$-projective boundedness) Let $\mathcal{Y}_{1}, \ldots, \mathcal{Y}_{n}$ be locally compact Hausdorff spaces, and define

$$
\|\mu\|_{p b_{\tau, n}}:=\sup \left\{\left\|\eta_{f ; \mu}\right\|_{\mathcal{V}_{n}\left(\mathcal{Y}_{1}, \ldots, \mathcal{Y}_{n}\right)}:\|f\|_{\mathcal{V}_{n}\left(\mathcal{X}_{1} \times \mathcal{Y}_{1}, \ldots, \mathcal{X}_{n} \times \mathcal{Y}_{n}\right)} \leq 1\right\} .
$$

We say that $\mu$ is $\tau$-projectively bounded if $\|\mu\|_{p b_{\tau, n}}<\infty$; the class of $\tau$ projectively bounded $\mathcal{F}_{n}$-measures on $\mathcal{X}_{1} \times \cdots \times \mathcal{X}_{n}$ is denoted by $\mathcal{P B} \mathcal{F}_{\tau, n}=$ $\mathcal{P B F} \mathcal{F}_{\tau, n}\left(\mathcal{A}_{1}, \ldots, \mathcal{A}_{n}\right)$.

2. ( $g$-projective boundedness) Let $\mathcal{X}_{1}, \ldots, \mathcal{X}_{n}$ be LCA groups. For $\mu \in$ $\mathcal{F}_{n}\left(\mathcal{A}_{1}, \ldots, \mathcal{A}_{n}\right)$ and elementary tensors $f=f_{1} \otimes \cdots \otimes f_{n} \in \mathcal{V}_{n}\left(\mathcal{X}_{1}, \ldots, \mathcal{X}_{n}\right)$, let

$\Psi_{f ; \mu}\left(y_{1}, \ldots, y_{n}\right)=\int f\left(x_{1}+y_{1}, \ldots, x_{n}+y_{n}\right) \mu\left(d x_{1}, \ldots, d x_{n}\right) \quad\left(y_{j} \in \mathcal{X}_{j}\right)$

and define

$$
\|\mu\|_{p b_{g, n}}:=\sup \left\{\left\|\Psi_{f ; \mu}\right\|_{\mathcal{V}_{n}\left(\mathcal{X}_{1}, \ldots, \mathcal{X}_{n}\right)}:\|f\|_{\mathcal{V}_{n}}=\prod_{j}\left\|f_{j}\right\|_{\infty} \leq 1\right\} .
$$

We say that $\mu$ is $g$-projectively bounded if $\|\mu\|_{p b_{g, n}}<\infty$; the class of $g$ projectively bounded $\mathcal{F}_{n}$-measures on $\mathcal{X}_{1} \times \cdots \times \mathcal{X}_{n}$ is denoted by $\mathcal{P B F} \mathcal{F}_{g, n}=$ $\mathcal{P B \mathcal { F }}{ }_{g, n}\left(\mathcal{A}_{1}, \ldots, \mathcal{A}_{n}\right)$.

The symbols $\tau$ and $g$ in Definition 3 denote, respectively, topological and grouptopological projective boundedness. It is straightforward to check that $\mathcal{P B F} \mathcal{F}_{n}$, $\mathcal{P B \mathcal { F }} \mathcal{F}_{g, n}$, and $\mathcal{P} \mathcal{B} \mathcal{F}_{\tau, n}$ are Banach spaces. To verify that every $\mu \in \mathcal{P B} \mathcal{F}_{g, n}$ is a convolver, let $\nu \in \mathcal{F}_{n}\left(\mathcal{X}_{1}, \ldots, \mathcal{X}_{n}\right)$ be arbitrary and consider a linear form $\Lambda: \mathcal{V}_{n}\left(\mathcal{X}_{1}, \ldots, \mathcal{X}_{n}\right) \rightarrow \mathbf{C}$ given by

$$
\Lambda(f)=\int\left(\int f\left(x_{1}+y_{1}, \ldots, x_{n}+y_{n}\right) \mu\left(d x_{1}, \ldots, d x_{n}\right)\right) \nu\left(d y_{1}, \ldots, d y_{n}\right) .
$$


Then $\Lambda$ is bounded, and the $\mathcal{F}_{n}$-measure $\lambda$ representing this linear action satisfies $\hat{\lambda}=\hat{\mu} \hat{\nu}$. Each $\mu \in \mathcal{P} \mathcal{B F}_{g, n}$ can be identified with a bounded linear operator $T_{\mu}$ : $\mathcal{F}_{n} \rightarrow \mathcal{F}_{n}$ given by $T_{\mu}(\nu)=\mu * \nu$. By a routine argument, $\mu \in \mathcal{P} \mathcal{B} \mathcal{F}_{g, n}$ if and only if the domain of $T_{\mu}$ is $\mathcal{F}_{n}$, and thus $\mathcal{P B F} \mathcal{F}_{g, n}$ is precisely the space of convolvers.

For $\mu \in \mathcal{F}_{n}$, let $\mathcal{D}(\mu)=\left\{\alpha \in \mathcal{F}_{n}: \mu * \alpha\right.$ exists $\}$, i.e., $\mathcal{D}(\mu)$ is the domain of $T_{\mu}$. Results in [GS1] and [GS2] state that in a two-dimensional setting $\mathcal{D}(\mu)=\mathcal{F}_{2}$ for all $\mu \in \mathcal{F}_{2}$, and there exist $\mu \in \mathcal{F}_{3}$ with $\mathcal{D}(\mu) \varsubsetneqq \mathcal{F}_{3}$. Thus it is natural to ask: for $\mu \in \mathcal{F}_{2}\left(\sigma\left(\mathcal{A}_{1} \times \mathcal{A}_{2}\right), \mathcal{A}_{3}\right)$, is $\mathcal{D}(\mu)=\mathcal{F}_{3}\left(\mathcal{A}_{1}, \mathcal{A}_{2}, \mathcal{A}_{3}\right)$ ? We show that the answer is no.

Let $\mathcal{S}\left(\mathcal{A}_{j}\right)$ be the space of $\mathcal{A}_{j}$-simple functions on $\mathcal{X}_{j}$, equipped with the uniform norm. $\mathcal{V}_{n}\left(\mathcal{A}_{1}, \ldots, \mathcal{A}_{n}\right)$ will denote the completion of $\mathcal{S}\left(\mathcal{A}_{1}\right) \otimes \cdots \otimes \mathcal{S}\left(\mathcal{A}_{n}\right)$ with respect to the projective tensor norm.

Proposition 4. $\mathcal{P} \mathcal{B F}_{n} \subset \mathcal{P B \mathcal { F }} \mathcal{T}_{\tau, n} \subset \mathcal{P B F}_{g, n}$.

Proof. The second inclusion is immediate from the definitions. Let $\mathcal{X}_{1}, \ldots, \mathcal{X}_{n}$ be compact spaces. A theorem of Saeki [S] states that

$$
C\left(\mathcal{X}_{1} \times \cdots \times \mathcal{X}_{n}\right) \cap \mathcal{V}_{n}\left(\mathcal{A}_{1}, \ldots, \mathcal{A}_{n}\right)=\mathcal{V}_{n}\left(\mathcal{X}_{1}, \ldots, \mathcal{X}_{n}\right) .
$$

We show that

$$
\left\|\eta_{f ; \mu}\right\|_{\mathcal{V}_{n}\left(\mathcal{Y}_{1}, \ldots, \mathcal{Y}_{n}\right)} \leq\|f\|_{\mathcal{V}_{n}}\|\mu\|_{p b_{n}}, f \in \mathcal{V}_{n}\left(\mathcal{X}_{1} \times \mathcal{Y}_{1}, \ldots, \mathcal{X}_{n} \times \mathcal{Y}_{n}\right)
$$

(See (11) for the definition of $\eta_{f ; \mu}$.) Let $g=g_{1} \otimes \cdots \otimes g_{n}$ be an elementary tensor in $C_{c}\left(\mathcal{X}_{1} \times \mathcal{Y}_{1}, \ldots, \mathcal{X}_{n} \times \mathcal{Y}_{n}\right)$. (The subscript $c$ denotes compact support.) Then $\eta_{g ; \mu} \in$ $C_{c}\left(\mathcal{Y}_{1} \times \cdots \times \mathcal{Y}_{n}\right)$. For locally compact spaces $X$ and $Y, \mathcal{V}_{2}(X, Y)$ is uniformly dense in $C_{0}(X \times Y)$, and thus for $k=1, \ldots, n$ there exist sequences $\left\{\rho_{j k}\right\} \subset \mathcal{S}\left(\mathcal{A}_{k}\right) \otimes \mathcal{S}\left(\mathcal{B}_{k}\right)$ with $\lim _{j \rightarrow \infty} \rho_{j k}=g_{k}$ in the uniform norm. Let $\theta_{j}=\rho_{j 1} \otimes \cdots \otimes \rho_{j n}$. Then $\lim _{j} \eta_{\theta_{j} ; \mu}=\eta_{g ; \mu}$ (uniform limit), and $\left\{\eta_{\theta_{j} ; \mu}\right\}$ is a $\mathcal{V}_{n}\left(\mathcal{B}_{1}, \ldots, \mathcal{B}_{n}\right)$-Cauchy sequence satisfying

$$
\left\|\eta_{\theta_{j} ; \mu}\right\|_{\mathcal{V}_{n}\left(\mathcal{B}_{1}, \ldots, \mathcal{B}_{n}\right)} \leq\left\|\rho_{j 1}\right\|_{\infty} \cdots\left\|\rho_{j n}\right\|_{\infty}\|\mu\|_{p b_{n}} .
$$

By (44), $\eta_{g ; \mu} \in \mathcal{V}_{n}\left(\mathcal{Y}_{1}, \ldots, \mathcal{Y}_{n}\right)$. Let $\epsilon>0$, and choose $f \in \mathcal{V}_{n}\left(\mathcal{X}_{1} \times \mathcal{Y}_{1}, \ldots, \mathcal{X}_{n} \times \mathcal{Y}_{n}\right)$. Without loss of generality, we can take $f$ to be compactly supported. We represent

$$
f=\sum_{k} g_{k}
$$

where $g_{k}=g_{1 k} \otimes \cdots \otimes g_{n k}$, and

$$
\|f\|_{\mathcal{V}_{n}} \leq(1+\epsilon) \sum_{k}\left\|g_{1 k}\right\|_{\infty} \cdots\left\|g_{n k}\right\|_{\infty}
$$

with each $g_{k} \in C_{c}\left(\mathcal{X}_{1} \times \mathcal{Y}_{1}\right) \otimes \cdots \otimes C_{c}\left(\mathcal{X}_{n} \times \mathcal{Y}_{n}\right)$. Then $\eta_{f ; \mu}=\sum_{k} \eta_{g_{k} ; \mu}$, and (51) follows.

We do not know which of the inclusions in Proposition 4 are proper. $\left(\mathcal{P B F} \mathcal{F}_{n}\right.$ is properly contained in $\mathcal{P} \mathcal{B F}_{g, n}$, as shown by the 'fractional' forms in B3.)

We now show that in a three-dimensional setting, an $\mathcal{F}_{2}$-measure need not be a convolver.

Lemma 5. Let $\mathcal{X}_{1}, \mathcal{X}_{2}$, and $\mathcal{X}_{3}$ be infinite locally compact abelian groups with respective Borel fields $\mathcal{A}_{1}, \mathcal{A}_{2}$, and $\mathcal{A}_{3}$. For every $K>0$ there exists a discrete measure $\mu$ on $\mathcal{X}_{1} \times \mathcal{X}_{2} \times \mathcal{X}_{3}$ satisfying $\|\mu\|_{\mathcal{F}_{2}\left(\sigma\left(\mathcal{A}_{1} \times \mathcal{A}_{2}\right), \mathcal{A}_{3}\right)} \leq 1$ and $\|\mu\|_{p b_{g, 3}\left(\mathcal{A}_{1}, \mathcal{A}_{2}, \mathcal{A}_{3}\right)} \geq$ $K$. 
Proof. Let $\mathbf{Z}_{n}$ be the group of integers under addition modulo $n$, and let $\hat{\mathbf{Z}}_{n}$ be its dual. Let $\mu_{n}: C\left(\hat{\mathbf{Z}}_{n} \times \hat{\mathbf{Z}}_{n}\right) \times C\left(\mathbf{Z}_{n}\right) \rightarrow \mathbf{C}$ be given by

$$
\mu_{n}(f, h)=\sum_{m \in \hat{\mathbf{Z}}_{n}} \frac{f(m, m)}{\sqrt{n}} \hat{h}(m) .
$$

We show that $\left\|\mu_{n}\right\|_{\mathcal{F}_{2}\left(\hat{Z}_{n} \times \hat{Z}_{n}, Z_{n}\right)} \leq 1$ and $\left\|\hat{\mu}_{n}\right\|_{\mathcal{V}_{3}\left(Z_{n}, Z_{n}, \hat{Z}_{n}\right)} \rightarrow \infty$ as $n \rightarrow \infty$. (For convenience, we write $\mathcal{F}_{2}\left(\hat{Z}_{n} \times \hat{Z}_{n}, Z_{n}\right)$ for $\mathcal{F}_{2}\left(2^{\hat{Z}_{n} \times \hat{Z}_{n}}, 2^{Z_{n}}\right)$.) Clearly, $\left\|\mu_{n}\right\|_{\mathcal{F}_{2}} \leq 1$ (by Cauchy-Schwarz). $\mu_{n}$ is also an element of $\mathcal{F}_{3}\left(\hat{\mathbf{Z}}_{n}, \hat{\mathbf{Z}}_{n}, \mathbf{Z}_{n}\right)$ :

$$
\mu_{n}(f, g, h)=\sum_{m \in \hat{\mathbf{Z}}_{n}} \frac{f(m) g(m)}{\sqrt{n}} \hat{h}(m) .
$$

Then

$$
\begin{gathered}
\hat{\mu}_{n}(j, k, l)=\sum_{m \in \hat{\mathbf{Z}}_{n}} \frac{e^{-\frac{2 \pi i j m}{n}} e^{-\frac{2 \pi i k m}{n}}}{n \sqrt{n}} \sum_{s \in \mathbf{Z}_{n}} e^{\frac{-2 \pi i(l+m) s}{n}} \\
=\frac{1}{\sqrt{n}} e^{2 \pi i j l / n} e^{2 \pi i k l / n} .
\end{gathered}
$$

Define

$$
\Phi_{n}(j, k, l)=\frac{1}{n^{2}} e^{-2 \pi i j l / n} e^{-2 \pi i k l / n} .
$$

Let $\mathbf{s}, \mathbf{t}$, and $\mathbf{u}$ be elements in the unit ball of $\ell^{\infty}\left(\mathbf{Z}_{n}\right)$. Then

$$
\begin{gathered}
\left|\sum_{j, k, l} \Phi_{n}(j, k, l) \mathbf{s}(j) \mathbf{t}(k) \mathbf{u}(l)\right| \\
=\frac{1}{n^{2}}\left|\sum_{l} \mathbf{u}(l) \sum_{j} \mathbf{s}(j) e^{-2 \pi i j l / n} \sum_{k} \mathbf{t}(k) e^{-2 \pi i k l / n}\right| \\
=\left|\sum_{l} \mathbf{u}(l) \hat{\mathbf{s}}(l) \hat{\mathbf{t}}(l)\right| \leq\|\mathbf{u}\|_{\infty}\|\hat{\mathbf{s}}\|_{2}\|\hat{\mathbf{t}}\|_{2} \leq\|\mathbf{s}\|_{\infty}\|\mathbf{t}\|_{\infty} \leq 1 .
\end{gathered}
$$

Thus, $\left\|\Phi_{n}\right\|_{\mathcal{F}_{3}} \leq 1$. However,

$$
\left|\sum_{j, k, l} \hat{\mu}_{n}(j, k, l) \Phi_{n}(j, k, l)\right|=\sqrt{n} .
$$

Therefore, by the duality $\left(\mathcal{V}_{3}\right)^{*}=\mathcal{F}_{3}$,

$$
\sqrt{n} \leq\left\|\hat{\mu}_{n}\right\|_{\mathcal{V}_{3}} \leq\left\|\mu_{n}\right\|_{p b_{3}} .
$$

Let $[N]=\{1, \ldots, N\}$. Given $K>0$, there exist $N$ and $\mu \in \mathcal{F}_{2}\left([N]^{2},[N]\right)$ with $\|\mu\|_{\mathcal{F}_{2}} \leq 1$ and $\|\mu\|_{p b_{3}}>K$. If $\mu=\left\{\mu_{x y z}:(x, y, z) \in[N]^{3}\right\}$, there exist arrays $a, b, c$ in the unit ball of $\ell^{\infty}\left([N]^{2}\right)$ such that

$$
\left\|\eta_{a, b, c ; \mu}\right\|_{\mathcal{V}_{3}([N],[N],[N])}>K,
$$

where

$$
\eta_{a, b, c ; \mu}(i, j, k)=\sum_{(x, y, z) \in[N]^{3}} \mu_{x y z} a_{x i} b_{y j} c_{z k}, \quad(i, j, k) \in[N]^{3} .
$$


Let $F_{m}$ and $G_{m}$ be disjoint mutually independent subsets of $\mathcal{X}_{m}$ of cardinality $N$ given by

$$
F_{m}=\left\{s_{j m}: j \in[N]\right\}, G_{m}=\left\{t_{j m}: j \in[N]\right\}, \quad m=1,2,3 .
$$

(Disjoint subsets $A$ and $B$ of an abelian group are mutually independent if given elements $\left(a_{1}, b_{1}\right)$ and $\left(a_{2}, b_{2}\right)$ of $A \times B$, the relation $a_{1}+b_{1}=a_{2}+b_{2}$ implies that $a_{1}=a_{2}$ and $b_{1}=b_{2}$.) Define a measure $\tilde{\mu}$ on $\mathcal{X}_{1} \times \mathcal{X}_{2} \times \mathcal{X}_{3}$ by

$$
\tilde{\mu}=\sum_{(x, y, z) \in[N]^{3}} \mu_{x y z} \delta_{s_{x 1}} \otimes \delta_{s_{y 2}} \otimes \delta_{s_{z 3}},
$$

and observe that $\|\tilde{\mu}\|_{\mathcal{F}_{2}\left(\sigma\left(\mathcal{A}_{1} \times \mathcal{A}_{2}\right), \mathcal{A}_{3}\right)} \leq 1$. Therefore, by the independence of $F_{m}$ and $G_{m}$, we can find $f \in C_{0}\left(\mathcal{X}_{1}\right), g \in C_{0}\left(\mathcal{X}_{2}\right)$, and $h \in C_{0}\left(\mathcal{X}_{3}\right)$ with

$$
f\left(s_{i 1}+t_{j 1}\right)=a_{i j}, \quad g\left(s_{i 2}+t_{j 2}\right)=b_{i j}, \quad h\left(s_{i 3}+t_{j 3}\right)=c_{i j},
$$

so that

$$
\eta_{a, b, c ; \mu}(i, j, k)=\Psi_{f \otimes g \otimes h ; \tilde{\mu}}\left(t_{i 1}, t_{j 2}, t_{k 3}\right), \quad(i, j, k) \in[N]^{3} .
$$

(Refer to (1) and (2) for definitions of $\eta$ and $\Psi$.) Thus $\left\|\Psi_{f \otimes g \otimes h ; \mu}\right\|_{\mathcal{V}_{3}} \geq K$, and hence $\|\tilde{\mu}\|_{p b_{g, n}} \geq K$.

Corollary 6. If the underlying $\sigma$-algebras $\mathcal{A}_{1}, \mathcal{A}_{2}$, and $\mathcal{A}_{3}$ are infinite, there exists $\mu \in \mathcal{F}_{2}\left(\sigma\left(\mathcal{A}_{1} \times \mathcal{A}_{2}\right), \mathcal{A}_{3}\right)$ which is not a convolver in $\mathcal{F}_{3}$.

\section{TWO FURTHER QUESTIONS}

1. It is shown in [B3] that scalar measures are projectively bounded. Hence, by Proposition 4 all scalar measures are convolvers. An $\mathcal{F}_{3}$-measure $\mu$ is an $\mathcal{F}_{\frac{3}{2}}{ }^{-}$ measure if, when any one coordinate is fixed, $\mu$ extends to a scalar measure in the remaining two coordinates. (See [B2] for details.) We have the proper containments $\mathcal{F}_{1} \varsubsetneqq \mathcal{F}_{\frac{3}{2}} \varsubsetneqq \mathcal{F}_{2}$. We do not know whether all $\mathcal{F}_{\frac{3}{2}}$-measures are convolvers.

2. Let $\mathcal{X}_{1}, \ldots, \mathcal{X}_{n}$ be LCA groups. It is shown in [ZS] that the space of completely bounded $n$-linear forms on $C_{0}\left(\mathcal{X}_{1}\right) \times \cdots \times C_{0}\left(\mathcal{X}_{n}\right)$ has a natural Banach *-algebra structure extending that of $\mathcal{F}_{1}$ on $\mathcal{X}_{1} \times \cdots \times \mathcal{X}_{n}$. We do not know whether all completely bounded forms are convolvers, or if all convolvers are completely bounded.

\section{REFERENCES}

[B1] R. Blei, Fractional dimensions and bounded forms, Mem. Amer. Math. Soc. 57 (1985), no. 331. MR 87k:26021]

[B2] An extension theorem concerning Fréchet measures, Canad. Math. Bull. 38 (1995), 278-285. MR 96k:28007

[B3] _ Projectively bounded Fréchet measures, Trans. Amer. Math. Soc. 348 (1996), 44094432. MR 97a:28009

[GS1] C. C. Graham and B. M. Schreiber, Bimeasure algebras on LCA groups, Pacific Jour. Math. 115 (1984), 91-127. MR 86a:43003 
[GS2] _ Projections in spaces of bimeasures, Canad. Math. Bull. 31 (1988), 19-25. MR 89b:43004

[S] S. Saeki, The ranges of certain isometries of tensor products of Banach spaces, J. Math. Soc. Japan, 23 (1971), 27-39. MR 46:7924

[ZS] G. Zhao and B. M. Schreiber, Algebras of multilinear forms on groups, Contemp. Math. 189 (1995), 497-511. MR 96i:43001

Department of Mathematics, University of Connecticut, Storrs, Connecticut 06269

E-mail address: Blei@uconnvm.uconn.edu

Department of Mathematics \& Computer Science, Arkansas State University, Box 70, State University, Arkansas 72467

E-mail address: Caggiano@csm.astate.edu 